\title{
MRI of shoulder instability: Review article
}

\section{Hamdan Abdelhameed Aly ${ }^{\mathrm{a}}$, Mohammad Hassan Alkousy ${ }^{\mathrm{a}}$, Khaled Fawzy Zaky Mohammad Tharwat Mahmoud Solymana}

\section{a Department of Radio-diagnosis, Faculty of Medicine, Sohag University, Sohag, Egypt.}

Background: Glenohumeral (GH) instability is a common entity that primarily affects young active populations and athletes in particular. Shoulder dislocation occurs when the humeral head is forced out of the glenoid fossa. Once the soft tissues supporting the shoulder become loose or torn, the shoulder joint becomes prone to dislocations. Various imaging techniques are available for the evaluation of shoulder joint abnormalities including plain radiographs, conventional arthrography, ultrasonography, computed tomography, MRI and MR arthrography. Magnetic resonance imaging is considered the best modality for assessment of shoulder joint pathology as it provides superior multiplaner capability and exceptional soft tissue contrast. MR arthrography, in particular, is more superior to conventional (non-arthrographic) MRI for the detection of the assessment of labral tears and articular cartilage damage. We have searched literatures in PubMed, Google scholar, Egyptian bank of knowledge and science direct.

Conclusion: Various imaging techniques are useful in assessment of shoulder instability. MRI and MR arthrography are considered the imaging modalities of choice for such cases as they provide superior soft tissue contrast in addition to the benefit gained form joint distension in MR arthrography allowing for better assessment of the labrum.

Keywords: Shoulder instability; MR Imaging; MR arthrography; Labrum; Glenohumeral ligaments.

\section{Introduction}

The shoulder joint is an imperfect ball and socket joint where the glenoid (socket) is smaller than the head of the humerus (the ball) and at the same time it has a larger radius of curvature. This anatomy provides the joint with exceptional mobility at the expense of stability (Pastor et al., 2014).

Radiology plays a crucial role in the evaluation and management of glenohumeral instability. MRI studies of the shoulder comprise both non arthrographic (conventional MRI) and MR arthrographic techniques. Conventional MRI allows evaluation of the shoulder as a whole, while MR arthrography provides joint distension allowing for better evaluation of the labrum and ligaments as well as better detection of partial thickness rotator cuff tears (RCTs) which makes it the imaging modality of choice in assessment of shoulder instability.

\section{Instability patterns}

2.1. Anterior Instability: The shoulder joint is the most commonly dislocated joint in the body. Most dislocations (90\%) are directed anteriorly. The shoulder is usually forced anteriorly in abduction, external rotation, and extension at the time of dislocation. The 
patient sometimes can't realize that the shoulder has been dislocated and relocated spontaneously. Unless confirmed through reduction maneuvers or radiographs, $1^{\text {st }}$ time dislocations can be hard to diagnose. After traumatic shoulder dislocation (treated non operatively), the likelihood of recurrent shoulder dislocation depends on patient age and the date of initial injury. Young patients (less than 20 years) have an increased risk of recurrent dislocation (more than 90\% including subluxation events) than those older than 40 years (less 10\%) (Gupta et al., 2018).

2.2. Posterior shoulder instability: Posterior dislocations are much less frequent than anterior shoulder dislocations and account for about $10 \%$ of all cases of shoulder instability. Posterior instability is difficult to diagnose clinically, and can be overlooked. Patients usually present with a vague shoulder pain, weakness, with or without joint clicking, and rarely have a clinical history of an acute traumatic event associated with the onset of symptoms. Hence, imaging plays a crucial role in the detection of abnormalities of the posterior capsulolabral complex and bony/soft tissue abnormalities that reduce joint stability. MR arthrography (MRA) is essential to reveal subtle labroligamentous findings (Albano et al., 2020).

2.3. Multi directional instability (MDI): It represents a complex condition without consistent diagnostic and treatment criteria. MDI often develops in the absence of trauma (atraumatic instability), involves both shoulders, and indicates joint hypermobility from generalized capsular laxity.

\section{General considerations for imaging shoulder instability}

3.1. Radiography: Radiographs (Figure 1) are useful for the initial work-up of shoulder instability. Despite using ultrasound and MRI as a first line of investigation in cases of shoulder pain on a wide scale, yet conventional radiography is still regarded as an essential imaging tool which provides a general overview of the shoulder anatomy and to rule-out serious conditions, such as tumors or arthritis. At least three views (two of which are orthogonal) are recommended in the setting of shoulder trauma. Anterior dislocation is going to be apparent in the AP view, but posterior dislocation can be missed at the hands of inexperienced radiologist (Brelin and Dickens, 2017). 

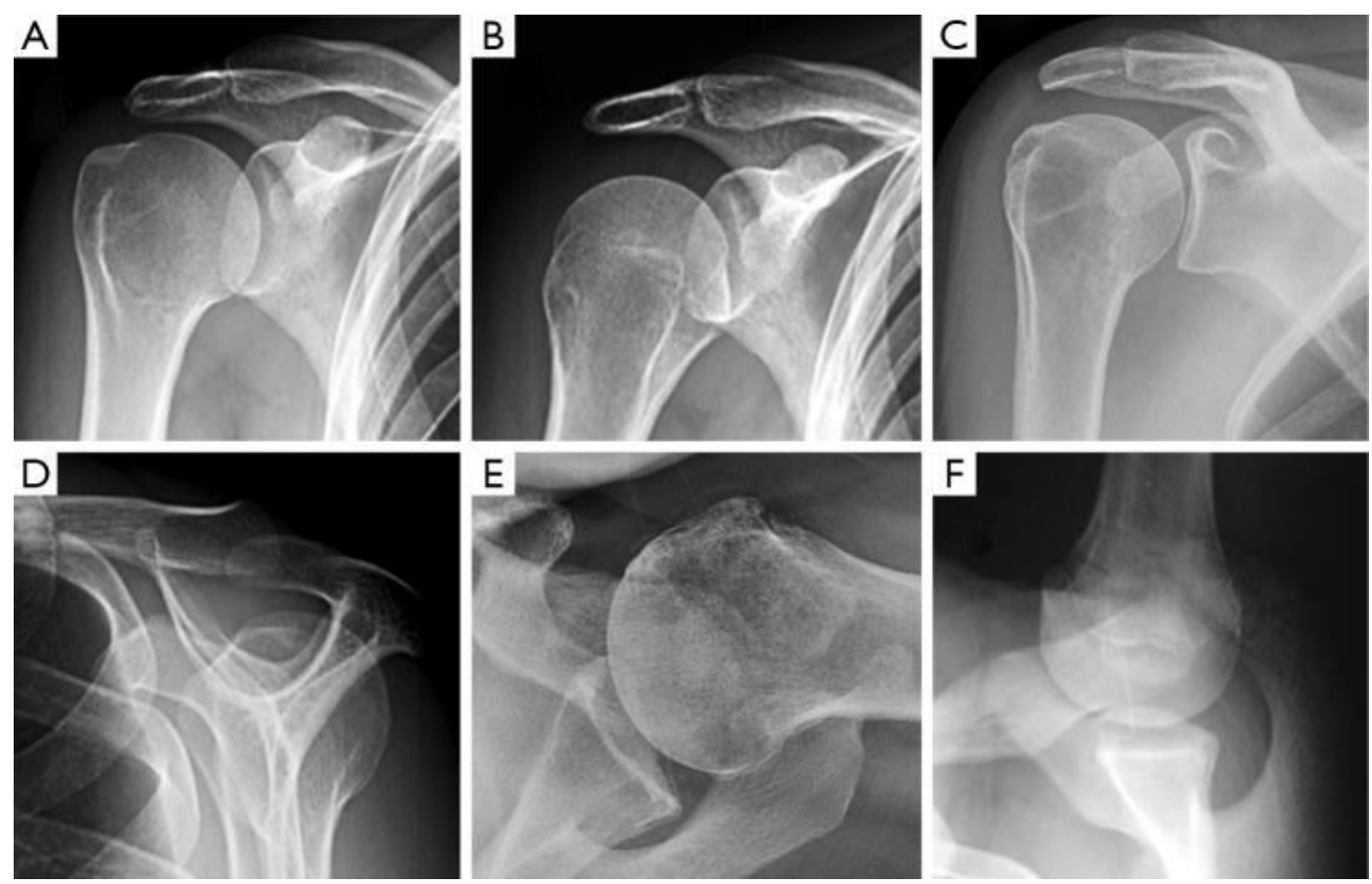

Fig.1. Radiographs in shoulder instability. (A) A-P shoulder radiograph in external rotation; (B) A-P shoulder radiograph in internal rotation; (C) Grashey glenoid profile view; (D) scapular Y view; (E) axillary view; (F) Bernagueau glenoid profile view. AP, anteroposterior (Quoted from Saba \& De Filippo, 2017).

\subsection{Multidetector computed tomography}

(MDCT): CT is usually performed after traumatic events to the shoulder in order to rule out fractures which are not detected on plain films or to assess the extent and severity of fractures already depicted in previous radiographs. CT arthrography (CTA) has also been shown to be accurate in detection of capsulolabral, ligamentous, and cartilaginous injuries as well as osseous injuries related to shoulder instability (sensitivities ranging from $82 \%$ to $100 \%$ and specificities ranging from 96\% to $100 \%$ ) (Acid et al., 2015)

3.3. MRI: MRI is the modality of choice in the evaluation of shoulder instability with MR arthrography being regarded as the goldstandard imaging technique for assessment of shoulder instability. The injection of paramagnetic contrast solution into the joint cavity allows for distension of the joint capsule making visualization of intraarticular structures better. The role of the radiologist in shoulder instability is localization of the lesions, revealing which one of the stabilizing elements is compromised, and to determine the acuity of lesions. Associated lesions have to be carefully reported as well (Sconfienza et al., 2018).

\section{MRI technique}

A dedicated phased array shoulder coil should be used with the patient lying supine and the arm placed in neutral or slightly external rotation position. Axial plane is obtained first with the field of view (FOV) including the acromion, entire humeral head and scapula. 
Coronal images should be angled along the oblique-coronal axis, in order to see the rotator cuff muscles and their tendons in continuity. Sagittal oblique plane is then selected perpendicular to the obtained coronal oblique images and parallel to the glenoid rim. Image should be obtained from the deltoid muscle laterally extending medially to the level of the scapular neck (Llopis et al., 2015).

Different pulse sequences including fluid-sensitive sequences with long TR and moderate to long TE (such as PD and T2WI) should be used and preferably with fat saturation. A sagittal T1WI images are recommended to assess for fatty atrophy of the rotator muscles as well as to assess the bone marrow. Additional positions such as abduction and external rotation (ABER) can be performed (Chen et al., 2008).

\section{MR arthrography technique}

Although high resolution conventional MRI has proved to have a high sensitivity for the detection of labral tears, direct MR arthrography with injection of contrast solution inside the joint cavity has gained popularity recently due to its ability to distend the joint capsule allowing for separation of the intra articular structures and outlining capsulolabral structures as well as the undersurface of the rotator cuff (RC) muscles (Steinbach, 2008)

'Direct' MR arthrography is a two step technique in which injection of the contrast solution into the joint cavity is performed first, and then the patient is transferred to the MRI scanner for imaging. The type of equipment used to guide the operator in performing direct arthrography relies on experience of the subject performing the injection as well as the availability of equipments. Injection in direct MR arthrography can be performed with palpation, fluoroscopy, ultrasonography (US), CT or MRI guidance. Fluoroscopic guided shoulder injection; however, remains the most widely used imaging guidance for shoulder injection with both anterior and posterior approaches (Rhee et al., 2012).

In all techniques of injection, a 20- or 22gauge 3.5 -inch $(0.8 \times 50-\mathrm{mm}$ beveled $)$ spinal needle was used to puncture the glenohumeral joint. US-guided procedure has the advantage of not exposing the patients or the radiologist to ionizing radiation when compared to Fluoroscopy-guided procedure and is also relatively simple and causes no patient discomfort as well as less time consuming (Gokalp et al., 2010). Up to $12 \mathrm{ml}$ of the diluted contrast solution is usually injected into the joint. Iodinated contrast can be added to this mixture in order to be able to visualize the contrast within the joint during fluoroscopic guided injection.

Protocols for MR arthrography vary widely. T1-weighted images with fat suppression are obtained in axial, coronal and sagittal planes to allow for evaluation of the capsulolabral structures. Fast spin echo PD or T2WI images in coronal and sagittal planes are also helpful 
for detection of bursal sided rotator cuff tears, para labral cysts, bursitis, bone marrow and muscle for contusions or strains. T1-weighted sagittal oblique sequence is also obtained to assess for fatty infiltration of muscles and marrow assessment as well. The quality of the rotator cuff muscles is an important determining factor in the prognosis and treatment of rotator cuff tears.

\section{Common lesions associated with anterior instability}

6.1. Classic soft Bankart lesion (The most typical and frequent lesion): Detachment of the anteroinferior labroligamentous structures from the glenoid with disruption of the scapular periosteum is termed classic Bankart lesion (Fig. 2). The interposition of the injected contrast solution or joint fluid in between the detached labrum and the underlying glenoid, allows for diagnosis by MR in $80 \%$ of the cases (Waldt et al., 2005).

Sometimes, the completely detached labroligamentous complex may migrate from its normal position, usually to a superior position and with time, this displaced labral tissue may undergo scarring forming an ovoid mass, known as glenoid labrum ovoid mass (GLOM) and shouldn't be confused for an intra articular loose body (De Conick et al., 2016).

\subsection{Anterior ligamentous periosteal sleeve} avulsion (ALPSA): The anteroinferior labrum is detached from the glenoid; however the scapular periosteum remains intact. As a result, the labrum is displaced medially against the glenoid neck. At MR arthrography, gadolinium should be seen extending in between the detached medially displaced labrum and the glenoid rim. Imaging the in the ABER position helps better visualization of such lesion.

6.3. Perthe's lesion: In Perthe's lesion, the anteroinferior labrum is detached, but again with intact scapular periosteum and the labrum lies in its normal position instead of being displacing medially. Subtle tracking of the injected contrast between the glenoid and pseudo-normal appearing labrum may be seen and is, again, best depicted in the ABER position.

\subsection{Glenolabral articular disruption (GLAD lesion):}

GLAD lesion involves a superficial tear to the anteroinferior labrum with an associated injury to the articular cartilage. The labrum is held firmly in place as the anterior ligamentous complex and the scapular periosteum remain intact, and therefore there is no instability. As such, GLAD lesion should be considered in patient complaining of persistent shoulder pain with no clinical evidence of rotator cuff tears, impingement, tendinosis or instability. 


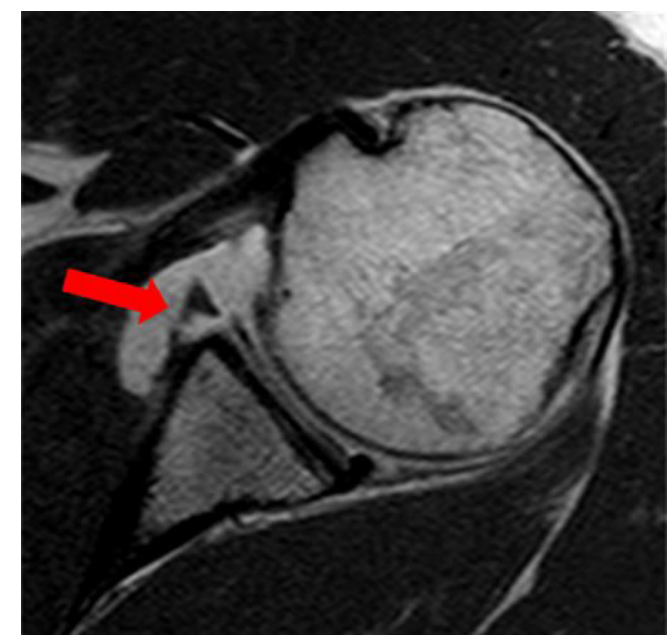

7. Common lesions associated with posterior instability

\subsection{Reverse Bankart Lesion- Reverse}

Bankart lesion is usually seen in patients who have sustained a substantial traumatic posterior shoulder dislocation. (Shah and Tung, 2009). A reverse Bankart lesion is a detachment of the postero-inferior labrum from the glenoid associated with disruption of the posterior scapular periosteum, typically
Fig. 2. A 26 Y-old patient with anterior shoulder instability. Axial T1WI MR arthrogram shows detached anteroinferior labrum with a disrupted scapular periosteum consistent with Bankart lesion (arrow) (Quoted from Saba \& De Filippo, 2017).

between the 6-O'clock and 10-O'clock positions (Fitzpatrick et al., 2016). MR arthrography shows contrast material extending into the substance of the posterior labrum or deep to the posterior labrum in association with a disrupted scapular periosteum (Fig.3). Sometimes, contrast material may be seen outlining the medial aspect of the posterior glenoid neck (Zlatkin and Sanders, 2013)

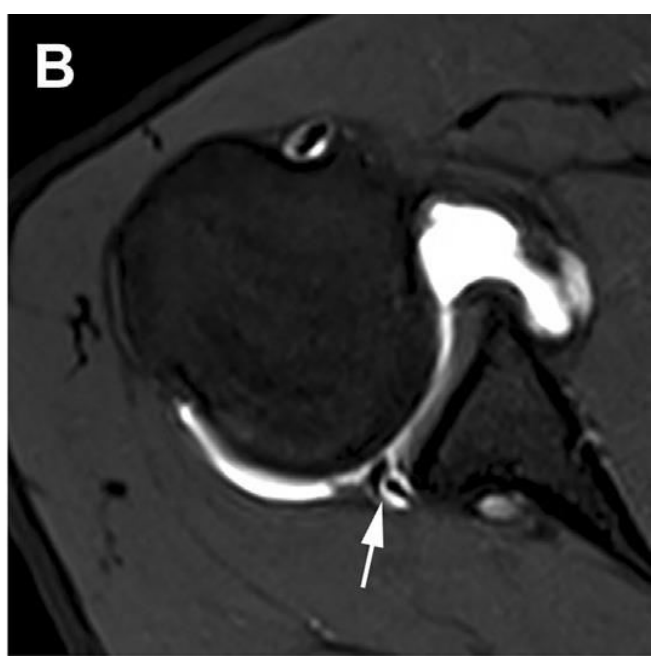

Fig.3. (A) Axial T1WI and (B) Axial T1-weighted with fat suppression imagesof the righ shoulder in a patient with post traumatic shoulder pain show a reverse Bankart lesion seen asa detached posteroinferior labrum with disrupted scapular periosteum (arrows) (Quoted from Albano et al., 2019). 


\subsection{Reverse bony Bankart: It represents} avulsion fracture of the posteroinferior glenoid during posterior shoulder dislocation. Posterior dislocation can also result in an impaction fracture of the lesser tuberosity, known as a reverse Hill-Sachs or McLaughlin lesion, the latter is associated with increased risk of instability when more than $30 \%$ of articular surface is involved or in the presence associated injuries of the posterior capsulolabral ligamentous complex (Cho et al., 2012).

\subsection{POLPSA Lesion-A POLPSA lesion is} the counterpart to ALPSA lesion. In POLPSA lesion, the posteroinferior labrum is detached from the glenoid along with intact medially displaced posterior scapular periosteum (Fig.4) (Chung and Steinbach, 2010). Disruption of the posterior capsule and scapular periosteum in combination with detached posteroinferior labrum helps differentiate it from posterior Bankart lesion. Sometimes, periosteum sleeve may be filled with fibrous tissue making no communication of contrast material visible between the lesion and the joint. at MR arthrography when fibrous tissue is present in the periosteal sleeve. The surgical procedure used in repairing POLPSA lesion differs from that in reverse Bankart lesion because the periosteal sleeve must be reduced before the labrum can be reattached (Fitzpatrick et al., 2016)
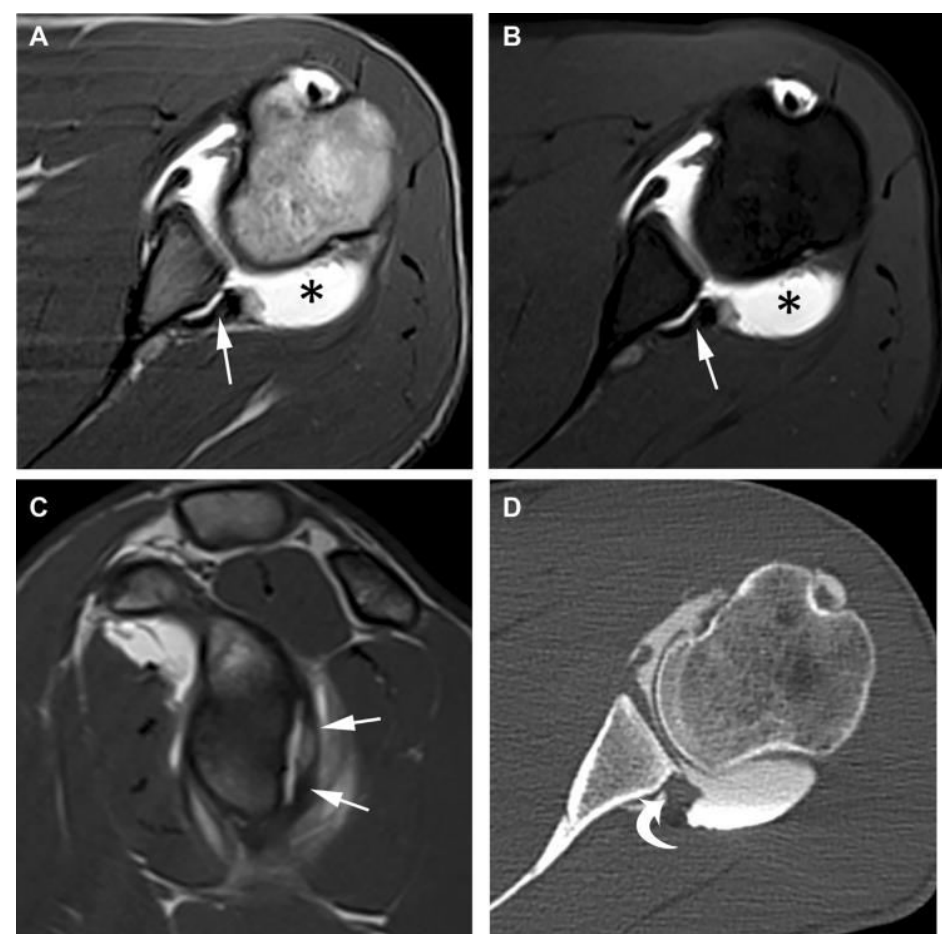

Fig.4. MR and CT arthrography of the left shoulder in a 22-year-old male with posterior shoulder instability. (A) Axial T1WI, (B) axial T1WI with fat suppression, and (C) sagittal T1WI MRA images show stripping of the posteroinferior labrum with intact periosteum (arrows) consistent with POLPSA lesion. Also note the pathologic increase of the axial area of the posterior capsular pocket (A, B, asterisk). (D) Axial CT arthrography performed 1 month later shows the periosteal sleeve filled by fibrous tissue (curved arrow). Quoted from Albano et al., 2020. 


\subsection{Posterior GLAD Lesion - This lesion is} similar to its anterior variant in which the posteroinferior glenoid is involved. It has proposed that the impaction between the posteroinferior glenoid and the internally rotated humeral head causes the posterior GLAD lesion resulting in posterior shoulder instability. MR imaging demonstrates focal defect at the articular cartilage between the 7O'clock and 9-O'clock positions, associated with superficial tear at the posteroinferior labrum (De Filippo et al., 2020).

\section{Conclusion}

Imaging plays accrual role in the investigation of shoulder pathology, especially that many of the clinical manifestations are not specific to certain pathology. Plain radiography and ultrasound can be used as primary investigation tools, while MRI is very useful for assessment of the shoulder as a whole, and MR arthrography in particular is useful in the assessment of shoulder instability and SLAP tears.

\section{References}

1. Pastor MF, Smith T, Struck M, Wellmann M. (2014). Stability versus mobility of the shoulder. Biomechanical aspects in athletes. Orthopade, 43 (3):209-214.

2. Gupta A, Gupta H. (2018). The radiological assessment of shoulder pathology. Orthopaedics and Trauma, 32(3): 144-152.

3. Albano D, Messina C, Sconfienza LM (2020). Posterior Shoulder Instability. Magnetic Resonance Imaging. Clinics of North America, 28 (2): 211-228.
4. Brelin A, Dickens JF (2017). Posterior Shoulder Instability. Sports Medicine and Arthroscopy Review, 25(3), 136143.

5. Acid S, Le Corroller T, Aswad R, Pauly V, Champsaur $P$ (2015). Preoperative imaging of anterior shoulder instability: diagnostic effectiveness of MDCT arthrography and comparison with MR arthrography and arthroscopy. American Journal of Roentgenology, 198 (3): 661-667.

6. Sconfienza LM, Albano D, Messina C, Silvestri E, Tagliafico AS (2018). How, when, why in magnetic resonance arthrography: an International Survey by the European Society of Musculoskeletal Radiology (ESSR). Eur Radiol, 28 (23): 56-68.

7. Llopis E, Montesinos P, Guedez M, Aguilella L, Cerezal L (2015). Normal Shoulder MRI and MR Arthrography: Anatomy and Technique. Seminars in Musculoskeletal Radiology, 19(3):212230.

8. Chen Q, Miller TT, Padron M, Beltran J. In: Pope T, Bloem JL, Beltran J, Morrison W, Wilson D, eds (2008). Imaging of the Musculoskeletal System. Philadelphia, PA: WB Saunders; 2008:101-125.

9. Steinbach LS (2008). MRI of shoulder instability. European Journal of Radiology, 68 (1), 57-71.

10. Rhee RB, Chan KK, Lieu JG, Kim BS, Steinbach LS (2012). MR and CT arthrography of the shoulder. Seminars in musculoskeletal radiology, 16 (1): 314.

11. Gokalp G., Dusak A., \& Yazici Z. (2010). Efficacy of ultrasonographyguided shoulder MR arthrography using a posterior approach. Skeletal Radiology, 39(6): 575-579.

12. Waldt S, Burkart A, Imhoff AB, Bruegel M, Rummeny EJ, Woertler K (2005). Anterior shoulder instability: accuracy of MR arthrography in the 
classification of anteroinferior labroligamentous injuries. Radiology, 237(2): 578-583.

13. De Coninck T, Ngai SS, Tafur M, Chung CB (2016). Imaging the Glenoid Labrum and Labral Tears. RadioGraphics, 36(6): 1628-1647.

14. Saba L, De Filippo M (2017). MR arthrography evaluation in patients with traumatic anterior shoulder instability. Journal of Orthopaedics, 14(1): 73-76.

15. Shah N, Tung GA (2009). Imaging signs of posterior glenohumeral instability. AJR Am J Roentgenol; 192(3):730-735.

16. Fitzpatrick D, Grubin J (2016). Navigating the alphabet soup of labroligamentous pathology of the shoulder. Am J Orthop; 45(2):58-60.

17. Zlatkin MB, Sanders TG (2013). Magnetic resonance imaging of the glenoid labrum. Radiol Clin North Am; 51(2): 279-297.

18. Cho JH, Chung NS, Song HK, Lee DH (2012). Recurrent posterior shoulder instability after rifle shooting. Orthopedics; 35 (11):670-679.

19. Chung C, Steinbach L (2010). MRI of glenohumeral instability. In: Chung $\mathrm{C}$, Steinbach L, eds. MRI of the upper extremity: shoulder, elbow, wrist and hand. Philadelphia, Pa: Wolters Kluwer Health/Lippincott Williams \& Wilkins; 277-319.

20. De Filippo M, Schirò S, Sarohia D, Barile A, Saba L, Cella S, et al. (2020). Imaging of shoulder instability Skeletal Radiol. Oct; 49(10):1505-1523. 